\title{
Infecciones intrahospitalarias por serratia marcescens
}

DRES.: GUlLLERMO W. LEDERMANN *, EDUARDO DONOSO **. MARCELLE JACOB *** y SRA. M. EliANA BLSTOS **.

Para un buen número de médicos, Serratia marcescens es sólo el inofensivo bacilo cuyo pigmento rojo le hiciera protagonizar una serie de anécdotas históricas (la "polenta sangrienta" de Legnaro, el pan y la hostia que sangran) (1) y que le valiera el epíteto de "prodigiosum" (chromobacterium, Erythrobacillus). Para otros, es el causante del "sindrome del pañal rojo" $(2,3)$.

Durante mucho tiempo se le estimó no patógeno, al extremo de utilizarlo como indicador en infecciones experimentales en voluntarios, sobresaliendo en este sentido las bacteremias provocadas por Burket (4) y McEntegart (5), quienes lo inoculaban en las encías antes de extracciones dentales, y las traqueitis con aerosoles de Paine (6) en estudios de contaminación ambiental. Sin embargo, después de un primer intento para relacionarlo con cuadros patógenos a fines del siglo pasado (7), desde 1913 (8) у ел forma continuada, diversas publicaciones informaban de su papel etiológico en septicemias $(9,10)$, meningitis (11), otitis (12), endocarditis (13) e infecciones urinarias (14), si bien es preciso reconocer que las descripciones bacteriológicas consignadas en estos trabajos son discordantes, al punto de hacer dudar que todos los gérmenes descritos hayan verdaderamente correspondido a la especie $\mathbf{S}$. marcescens.

La estrecha identidad de este organismo con su pigmento ha sido causa fundamental de que estas publicaciones no sean más numerosas. Hasta que Ewing (15) no demostrara que el pigmento era un carácter secundario, presente sólo en una minotía (alrededor del $20 \%$ ) de las cepas, en la práctica el diagnóstico se hizo siempre por esta llamativa propiedad, y es muy posible que buen

\footnotetext{
* Hosp. L. CaJvo Mackenna, Servicio Infecciosos.

** Hosp. L. Calyo Mackenna, Lab. Bacleriologia.

** Instítuto Bacteriológico de Chile.
}

número de Serratia hayan sido erróneamente identificadas como alguna de las especies del grupo Klebsiella-enterobacter, ya que sư exacto diagnóstico requiere de reactivos no siempre disponibles en un taboratorio de hospital.

En la década del 60, el mismo Ewing, Clayton (16) y Wilkoswky (17), entre otros, llamaron la atención sobre la creciente aparición de Serratia no pigmentada en infecciones intrahospitalarias graves. Todos coincidían en señalar que eran fundamentalmente afectados pacientes sometidos a instrumentación (cateterismo, sondeo, djálisis), a intervenciones quirúrgicas o que habían recibido prolongado tratamiento antibiótico. En nuestro país, Paredes (18) ha publicado recientemente una revisión del problema en adultos; en niños, en el Hospital Calvo Mackenna hemos encontrado, desde fines de 1973, un total de 8 casos, de los que reseñamos tres de los más jlustrativos. Aproximadamente desde esa misma fecha, en la Colección de Cepas del Instituto Bacteriológico hemos recibido para identificación otras seis cepas provenientes de infecciones en otros centros hospitalarios.

\section{MATERIAL Y METODO}

\section{Casos clínicos}

C. N. S. Lactante de sexo femenino, de 3 meses de edad y $4.530 \mathrm{gr}$ de peso, enviada para corregir quirúrgicamente una hernia hiatal deslizante. Al mometto de ingreso se constata la existencia de bronconeumonia, tratada con penicilina sódica por 7 días, indicándose luego una penicilina benzatina de $600.000 \mathrm{U}$. Una vez recuperada, hace una diarrea con deshidratación, tratada, entre otras medidas, con gentamicina $12 \mathrm{mgr} c / 12 \mathrm{hrs}$. I. M., combinada con 4 mgr. en sol. oral c/6 hrs. Su- 
perada esta nueva emergencia, se hace la hernia y piloroplastía. En el post-operatorio recibe ampicilina 100 mgr c/6 hrs. por 8 días, al $5^{\circ}$ de los cuales hace dehiscencia de suturas y perítonitis, debiendo ser reintervenida. Tres días después y estando nuevamente bajo tratamiento con gentamicina, presenta un cuadro febril con gran compromiso del estado general, que hace pensar en septicemia, instalándose terapia con cloxacilina y kanamicina, tomando muestra previa para hemocultivo. Cinco dias más tarde y pensando en sepsis por Pseudomonas, se cambia a carbenicilina $(200 \mathrm{mgr} \mathrm{c} / 8$ hrs. IM), con to cual se obtiene rípida y favorable respuesta, al punto de mantener el tratamiento aunque el estudio de sensibilidá (método de difusión) revela que cl bacilo gram negativo aislado del hemocultivo es resistente a carbenicilina, gentamicina y ampicilina. Con posterioridad es identificado este germen como Serratia marcescens no pigmentada.

A. C. B.- R. N. prenaturo, de sexo femenino $y$ siete días de edad, trasladado desde otro hospitai cont los diagnósticos de l) Ictericia por defecto de conjugación (tratada con recambio sanguíneo); 2) Obstrucción intestinal? y 3) ¿Sepsis? El niño, de un peso de 1.600 gr., estaba en malas condiciones generales, ictérico, febril, con progresivas sigoos de obstrucción intestinal. El hemograma mostraba teucocitosis de $\mathbf{1 4 . 0 0 0}$ con 5 baciloformes; la radiografía de abdomen sugería atresia intestinal alta, y el hemocultivo, informado con posterioridad al desenlace, fue positivo a Sertatia marcescens. Intervenido quirúrgicamente, se encuentra atresia yeyunal, que se corrige con anastomosis yeyuno-ileal. En el post-operatorio hace dehiscencia de suturas y peritonitis, debiendo ser reintervenido. $\mathrm{El}$ estado general empeora $y$ fallece al $11^{9}$ día de hospitalización. Durante tolo este tiempo y dexde tics días antes de su trastado, estuvo recibiendo penjeilina y gentarnicina. La autopsia reveló, aparte de la dehiscencia de suturas, focos hemorrágicos en meninges, pleura e hígado; condensación pulmonar y comunicación interauricular.

D. L.- Escolar de 9 años, de sexo femenino, con el antecedente de hidrocefalis secundaria a toxoplasmosis congénita, corregiđa a los 7 meses con la implanta. ción de una válvula derivativa ventrículo-auricular (Pudenz-Heyer). Se hospitaliza por presentar miocarditis $y$ hemorragia digestiva en el transcurso de segunda semana de fiebre tifoidea, comprobada con hemocultivo positivo a Salmonella paratyphi B, Widal $1 / 1000$ y hemograma concordante. Se trató con digitálicos, transfusiones sanguíneas y cloranfexicol $2 \mathrm{gr} / \mathrm{día}$, sin que cedicra la fiebre en 11 días de tratamiento, momento en que se observa edema en el trayecto del catéter valvalur en el cuello. Se hizo punción de la válvula, obteniéndose líquido turbio, en el que la tinción de Granı reveló la presencia de bacilos Gram negativos, pero cuyo cultivo no fuyo desarrollo microbiano. Pensando que este bacilo era la misma Salmonella, se hizo tratamiento intravalvular cun cloranfenicol por tres días. Ante la falta de resputesta a este antibiólico luego de 14 días de tratamiento, se cambio por sulfa-trimetoprim (50 mgr/kg-peso) con lo cual hubo mejoría del estado general y remisión de la curva térmica en seis días. De un mielocultivo practicado en el momento de cambiar tratamiento se aisló Serratia marcescens, germen que también se encontro en una nueva punción valvilar. Dada de alta a los 23 días de bospitalización, reingresa once dfas más tarde con signos de septicemia, aislándose Serratia de tres hemocultivos y de LCR obtenido por punción lumbar. Se trita durante un mes con gentamicina intramuscular (4 $\mathrm{mgr} / \mathrm{kg} / \mathrm{día})$, recibiendo simultáneamente durante 15 días un décimo de esta dosis intravalvular, puncionando la válvula cada 12 horas, con lo cual se logró buena mejoría y se normalizó el LCR.

Método bacteriológico. En todas las cepas se estudió la fermentación de glucosa, lactosa, sacarosa, maltosa, manitol, dulcitol, salicina, adonitol, inositol, sorbitol, arabinosa, rafinosa y trehalosa; la presencia de desoxiribonucleasa, ureasa, oxidasa, lisina-decarboxilasa y fenilalanina deaminasa; se verificó la reducción de nitratos, la utilización de citrato y de malonato de sodio; la producción de indol, de ácido sulfihidrico y la licuación de la gelatini. Se siguió el criterio de Edwards para diferenciar Serratia de Enterobacter y de Hafnia, fundamentando su diagnóstico en la producción de desoxiribonucleasa, licuación rápida de la gelatina, utilización del citrato, pero no del malonato de sodio, y la incapacidad para fermentar arabinosa y ramnosa. No se practicó serotipificacion, fagotipia ni se determinaron las bacteriocinas, por no disponer de los elementos necesarios. La sensibilidad antibiótica se determinó con el método de discos, midiendo la C. I. M. en tubos sólo para aquellos antíbióticos activos en placa.

RESULTADOS Y COMENTARIO. Las publicaciones más importantes sobre infecciones por Serratia $(19,20,21)$ coinciden en algunos puntos claves: son infecciones intrahospitalarias, en enfermos debilitados, generalmente con alguna grave enfermedad de fondo, tratados con antibióticos, sometidos a instrumentación o a intervenciones quirúrgicas. En todos ellos la Serratia, actuando como patógena oportunista y seleccionada por los antibióticos empleados, ha actuado como invasor secundario. Los tres casos clínicos que reseñamos a guisa de cjemplo, son los que mejor jlustran las condiciones referidas.

En el primero (C. N. S.) encontramos a Serratia marcescens provocando sepsis en un niño hospitalizado, que había recibido durante semanas una variada gama de antibióticos (penicilina, ampicilina, gentamicina) y que había sido intervenido quirúrgicamente.

En el segundo ejemplo (A. C. B.) el recién nicido había sicio sometido a recambio sanguíneo en un hospital, era prematuro y tenía una severa patología de fondo (Obstrucción intestinal por atresia). También había recibido antibióticos (pe- 
nicilina y gentamicina) desde su segundo día de vida. No consideramos la intervención quirúrgica, que fue posterior al hemocultivo desde donde se aisló el germen.

En el tercero, tenemos una infección intrahospitalaria que se inserta sobre una tifoidea complicada, favorecida por la presencia de una válvula artificial que, en la experiencia de los neurocirujanos, es de muy difícil esterilización. Aquí Ia "instrumentación" estaría representada por" la transfusión de sangre efectuada al ingreso: cxiste. también, un tratamiento antibiótico previo.

En cuanto a los cinco restantes casos, ellos reúnen, en mayor o menor grado. las mismas condiciones predisponentes.

En uno, la infección se instala en una traqueostomía practicada a una niña con una polineuritis ascendente; en otro. la Serratia provoca sepsis en un prematuro; en vías urinarias, aparece en niños con infecciones crónicas y tratamientos prolongados, como consecuencia de malformaciones.

De los casos presentados en otros hospitales, cuyas cepas recibimos en el Instituto Bacteriológico para su identificación, no disponemos de mayor información, ya que los cultivos que nos fueron referidos traian apenas los datos necesarios para orientar el diagnóstico bacteriológico. En todo caso, ellas nos indican aue el problema no es privativo de nuestro hospital.

Ignoramos si todos los casos presentados en un hospital fueron causados por la misma cepa. Esto puede determinarse estudiando tanto la producción como la sensibilidad a bacteriocinas. método que en "epidemias" intrahospitalarias ha permitido trazar el avance de éstas y, eventualmente, descubrir la fuente de infección $(22,23)$. Este método aparece como más ventajoso que la serotipificación o la fagotipia y es relativinuente sencillo $(24,25)$. La persistencia de una determinada cepa de Serratia en un hospital se comprueba también por la sensibilidad antibiótica. En un estudio realizado en tres hospitales de $N$. York (26) se vio que las cepas aisladas de un mismo hospital tenían un antibiograma similar. diferentc del antibiograma de cepas de otros centros. Los factores $\mathbf{R}$ eran también diferentes y trasmitían. en cada hospital, resistencia a distintos antibióticos, en uno para ampicilina, kanamicina, estreptomicina y sulfas, en otro, sólo para ampicilina. De nuestra pequeña casuística. ningún pattern ca-ç racterístico puede establecerse para cepas de un mismo hospital.

Aun cuando la mayoría de las publicaciones sobre Setratia indican a gentamicina como droga de elección $(27,28)$, nuestros resultados no son muy favorables a use antibiótico. (Tabla 1). Sin embargo, en el caso No 3 tuvinos buena respussta "in vivo", pese a que la C. I. M. estaba not encima de los $25 \mathrm{mcgr} / \mathrm{ml}$.
TABLA NO 1

SENSIBILIDAD IN VITRO DE SERRATIA MARCESCENS A NUEVE ANTIBIOTICOS Y QUIMIOTERAPICOS

\begin{tabular}{lccc}
\hline Antibiótico & No cepos & Sensibles & C I. M. en ugfml. \\
\hline Cloramfenicol & 12 & $2(16.6 \%)$ & 3.12 \\
Ampicilinat & 12 & 0 & - \\
S. de Colistio & 12 & 0 & - \\
Rifanicina & 12 & $10(83,3 \%)$ & $6.25-12.5$ \\
Gentanicina & 12 & $6(50 \%)$ & $0,10-12,5$ \\
Sulfametoxazol- & & & \\
trimetroprint. & 12 & $10(83,3 \%)$ & $3,12-25,0$ \\
Acido nulidixico & 12 & $12(100 \%)$ & $0.78-6.25$ \\
Nitrofurantoína & 12 & $11(91.7 \%)$ & no se determinó \\
Carbenicilina & 12 & $6(50 \%)$ & $0,78-50$ \\
\hline
\end{tabular}

Igualmente bueng fue la respuesta obtenida en ef primero de los enfermos con carbenicilina, recomendada como "Segunda opción" (29). Por lo demás la combinación de estos dos antibióticos se hat demostrado, in vitro e in vivo, como la mejor $(30,31)$ y podría constituir el tratrmiento de ataque mientras se efectúa el antibiograma, que debe hacerse de rutina en infecciones por este germen.

Rifamicina y sulfa-trimetoprim, pese a su C. I. M. alta, pueden constituir una alternativa en infecciones sistemáticas, $y$ en infecciones urinarias la nitrofurantoína, tan bucna in vitro como el ácido nalidixico. seria la droga de elección.

Creemos posible que el problema de infeccionos intrahospitalarias pueda ser mayor y que esté enmascarado por falta de un acertado diagnóstico de laboratorio, por to que estimamos indispensable ampliar los caracteres biológicos que se estudian de rutina, a fin de poder preparar Serratia de las especies que habitualmente se engloban bajo el término de "Klebsiella-Enterobacter".

Quizís estemos asistiendo a1 nacimicnto do la sueesora como "villana pigmentada" de Pseudomonas, y esta perspectiva tan poco halagiieña mueve a reflexionar otra vez sobre el uso indiscriminado $-y$ aparentemente inevitable- de los antibióticos.

\section{Resume.}

Se presentan ocho casos de infección intrahospitalaria por Serratia marcescens en un servicio pediatrico. Se recuerdan los factor's predisponentes: cirtigio, "instrumentecion", andibióticos $y$ grave enfermedad de fondo. Se estudia la sensibilidad a los antibióticos de las cepas aistadas, asi como stis caracteres bioquimicos, adviriendo que 
el pigmento rojo es un carácter secundario $e$ inconstante, cuya falta puede llevar a error diagnóstico con Klebsiello-Enterobacter, enmascarando asi la verdadera magnitud de la infección hospitalaria por este bacilo.

\section{SUMMARY}

Eight cases of intrahospitalary infection by Serratia marcescens were reported from a pediatric service. The authors entphasize the predisposing factors: surgery, "instrumentation", antibiotics and severe basic pathology. Antibiotic sensitjvity, as well as biochemical properties were studied, advising that red pigment is only a secondary and inconsistent character, whose failure can lead to a misleading diagnostic with Klebsiella-Enterobacter, thus masking the actual magnitude of Serratia's nosocomial infection.

\section{REFERENCIAS}

1.-Editorial. Infection due to Chromobacteria, JAMA., 148, 56, 1952.

2.-Waisman, H. A. and Stone, W. H. The presence of Serratia marcescens as the predominating organistr in the inestinal tract of the newborn: the occurrence of the "red diaper syndrome". Pediatrics, 21: $8-12,1958$.

3.- Hernández, R., Oyarce, P. y Arcaya, O. Sindrome del pañal rojo. Infección por Serratia marcescens. Rev. Chil. Ped.

4.-Burket, L. W. and Burn, C. G. Bacteremia following dental extraction: Demonstration of source of bacteria means of a non-pathogen (Serratia marcescens). J. Dent. Res,, 16:521-530, 1937.

5.-McEntegart, M. G. and Poterfield, J. S. Bacteremia following dental extractions. Lancet, 11: 596, 1949.

6,- Paine, T. F. Illness in man following inhalation of S. marcescens. J. Infect. Dis., 79: 226, 1946.

7.-Du Bois Saint-Sevrin. Panaris des pecheurs et microbe rouge de la sardine. Ann. Inst. Pasteur, 8: $152-160,1894$.

8.-Woodward, H. and Olash, $K$. A case of infection by the Bacterium prodigiosum. Lancet, i: 314 , 1913.

9.-Black, M. E. and Stuthan, J. Bacillus violacens infection in a human being. JAMA., 110: 1270 . 1938.
10.- Schattenberg, H. and Harris, W. H. A definite and unique occurrence of rapidly fatal infection caused by Bacillus violaceus Manilae. JAMA., 117: 2069, 1941.

11.-Aronson, J. D. and Alderman, I. The occurrence and bacteriological charactheristics of $\mathbf{S}$. marcescens from a case of meningitis. J. Bact., 46: 261, 1943.

12.- Cutitt, M., Sapuppo, R. Otite media crónica supurada por B. prodigiosus. Rev. paul. med., 39: 358-359, 1951 .

13.-Hawe, A.J. and Hughes, M. H. Bacterial endocarditis due to Chromobacterium prodigiosum. Brit. Med. J., I: 968, 1954.

14.-Constable, F. L. Urinary infection by Serratia marcescens. Brit. Med. J., 2: 110, 1955.

15.-Ewing, W. H., Davis, B. R. and Reavis. R. W. Studies on the Serratia group. C. D. C. Publ., Atlanta, Ga., 1959.

16.-Glayton, E. und Graevenitz, A. V. Non pigmented Serratia marcescens. JAMA, 197: 1059-1064, 1966.

17.-Wilkokske, C. J.; Washington, J. A.; Martin, W. J. and Ritts, R. E. Serratia marcescens: Biochemical characteristics, antibiotic susceptibility patterns and clinical significance. JAMA., 214: 2157-2161, 1970.

18.-Paredes, L. y col. Infecciones por Serratia marcescens. Estudio bacteriológico, antibiograma y significado clínico. Rev. Med. Chile., 102: 109, 1974.

19.- Modde, $\boldsymbol{H}$. Durch Serratia marcescens verursachte Krankheiten. Schweis. Med. Wschr., 102, 1386$1390,1972$.

20.-Crowder, J. G., Gilkey, G. H. and White, A. O. Serratia marcescens bacteremia: Clinical observations and studies of precipitin reactions. Arch. Int. Med., 128, 247, 1971.

21.-Ewing, W. H., Johnson, J. O. and Davis, B. R. The occurrencs of $\mathbf{S}$. marcescens in nosocomial infections. C. D. C. Publ., Atlanta, Ga., 1962.

22.-Traub, $W . H$. Epidemiological surveillance of Serratia marcecens infections by bacteriocin-typing. Appl. Microbiol., 22: 1058-1063, 1971.

23.- Tratb, W. H. Bacteriocin-typin of Setratia marcescens of known serotype group. Appl. Microbiol., 23: 979-981, 1972.

24.-Farmer, J. J. Epidemiological differentiation of Serratia marcescens: typing by bacteriocin production. Appl. Microbiol., 25: 218-225, 1972. 
25.-Farmer, J. J. Epidemiological differentiation of Serratia marcescens: typing by bacteriocin sensitivity. Appl. Microbiol., 23: 226-231, 1972.

26. - Schaefler, S. Winter $J$. et all. Specific distribution of a factors in $S$. marcescens isolated from hospital infections. Appl. microbiol., 22, 339-343, 1971.

27.-Wilfert, J. M. et all. Serratia marcescens: biochemical, serological and epidemiological characteristics and antibiotic susceptibility of strains isolated at Boston City Hospital. Appl. microbiol, 19: 345$350,1970$.
28.-Digout, G. McDonald S. and Van Rooyen, C. E. Sensitivity of Serratia to gentamicin and carbenicillin. N. S. Med. Bull. (Halirax), 50, 79-81, 1971.

29.- Thomton, G. F. and Andriole, V, T. Antibiotic sensitivities of non-pigmented Serratia marcescens to gentamicin and carbenicillin. J. Infect. Dis., 119, 393-394, 1969.

30.-Brisou, J. Comportement des Serratia d'origine hospitaliere en presence de quelques associations d'antitibiotiques. Path. biol., 19: 655-660, 1971.

31.-Farhoudi, H. D. Baubs, T. and Alí. N. Serratia marcescens endocarditis: succesful carbenicillin and gentamicin treatment. Ann. Int. Med., 78, 776, 1973. 